\title{
Model for Solute Redistribution During Rapid Solidification
}

\section{Citation}

Aziz, Michael J. 1982. Model for solute redistribution during rapid solidification. Journal of Applied Physics 53, no. 2: 1158-1168.

\section{Published Version}

http://dx.doi.org/10.1063/1.329867

\section{Permanent link}

http://nrs.harvard.edu/urn-3:HUL.InstRepos:2961250

\section{Terms of Use}

This article was downloaded from Harvard University's DASH repository, and is made available under the terms and conditions applicable to Other Posted Material, as set forth at http:// nrs.harvard.edu/urn-3:HUL.InstRepos:dash.current.terms-of-use\#LAA

\section{Share Your Story}

The Harvard community has made this article openly available.

Please share how this access benefits you. Submit a story.

\section{Accessibility}




\title{
Model for solute redistribution during rapid solidification
}

\author{
M.J. Aziz a) \\ Division of Applied Sciences, Harvard University, Cambridge, Massachusetts 02138
}

(Received 24 July 1981; accepted for publication 6 October 1981)

\begin{abstract}
A microscopic model for impurity uptake at a sharp crystal-liquid interface during alloy solidification is presented in terms of the bulk properties of the liquid and solid phases. The results for stepwise growth and continuous growth at the same interface velocity differ quantitatively but exhibit the same qualitative features. A transition from equilibrium segregation to complete solute trapping occurs as the velocity surpasses the diffusive speed of solute in the liquid. The location of the transition varies little with equilibrium segregation coefficient, and a kinetic limit to solute trapping is found to be quite unlikely. Comparison is made with other models; critical differences are pointed out. Coupled with a growth velocity equation and with macroscopic heat- and solute-diffusion equations, the model forms a complete description of one-dimensional crystal growth. The steady-state solution to this system is indicated for the case of a planar interface. The results are applied to describe regrowth from laser-induced melting. Preliminary comparison with experiment is made. The steady-state solution for thermal and impurity transport is suggested for use whenever detailed computer calculations are unavailable or are unnecessarily involved.
\end{abstract}

PACS numbers: 81.10.Fq, 64.75. $+\mathrm{g}$, 81.40.Ef, 79.20.Ds

\section{INTRODUCTION}

Until recently, descriptions of solidification of liquid alloys have focused on the near-equilibrium limit, in which the solidification speed is largely controlled by the rate of transport of latent heat away from the interface. In this case, crystal growth occurs slowly enough so that the solid and liquid immediately on either side of the interface can be considered to be.in local equilibrium; i.e., their compositions are given by the solidus and the liquidus on the phase diagram, and the only problem is determining the interface temperature. However, experiments on chill casting of molten alloys, ${ }^{1}$ on solidification of small, highly undercooled liquid droplets, and on thin molten surface layers created by pulsed laser irradiation have produced such rapid interface motion that the "local equilibrium" assumption can hardly be valid. In fact, in certain systems with retrograde solid solubility, splat quenching of molten alloys ${ }^{2}$ and regrowth at velocities $u \approx 1 \mathrm{~m} / \mathrm{s}$ from laser-induced melting ${ }^{3,4}$ have shown that substantial departures from equilibrium at the interface must exist. It was found that although the total free energy of the liquid dropped, as it must, during crystallization, the chemical potential of the minor component of dilute binary alloys often increased. Solute concentrations far in excess of the equilibrium solid solubility limit have been attained in this manner. The discovery of this phenomenon, termed "solute trapping," ruled out local equilibrium and the idea that the major and minor components act independently at the interface during rapid solidification. This behavior underscored a need for more comprehensive knowledge of interface structure and kinetics. In particular, the mechanism of and the limits to solute trapping, of interest to those who study atomic motions, have become important to technological applications of the newly-obtained enhanced solubilities.

\footnotetext{
${ }^{a}$ Allied Chemical Research Fellow.
}

A complete theory for the crystal-growth aspect of a one-dimensional binary solidification problem requires the simultaneous solution of four equations. There must be a model relating the composition of the growing solid to that of the liquid at the interface. There must be an expression relating the growth velocity to the local conditions at the interface (temperature, pressure, composition, etc.). Finally, there must be equations for transport of rejected solute and latent heat away from the interface. The simultaneous solution of these four equations tells us how the system will behave. The flow of heat away from the interface will cause an interfacial undercooling sufficient to drive the transformation at a velocity matching, in steady state, that of thermal escape. When growth is sufficiently slow, the interfacial undercooling is negligible and the entire process is heat flow limited. The second of the four equations may be ignored, the interface temperature may be taken to be the liquidus temperature, and the growth velocity may be found by solving the heat equation. However, if the temperature gradient becomes steeper, the growth velocity increases to a point where the finite interface mobility must be taken into account. The velocity-dependent composition of the growing solid and the composition of the liquid at the interface, determined by the diffusion equation, determine the driving force for the transformation after the interface temperature is known.

It is estimated ${ }^{5}$ that a transition to interface limited growth begins when the temperature profile decays to ambient at a decay length of approximately $60 \mu$. We expect both solidification of sufficiently small droplets and regrowth from laser annealing to be interface limited.

The basic idea behind solute trapping models is that solute atoms, in order to avoid being engulfed by the growing solid, must diffuse ahead of the interface. Solidification, involving only short-range atomic rearrangements at the interface and no long-range diffusive motion, can proceed much 
more rapidly than solute diffusion. Consequently, when growth occurs with speeds $u$ exceeding the diffusive speed $D_{L} / \lambda$, where $\lambda$ is the interatomic spacing, and $D_{L}$ is the solute diffusivity in the liquid, solute atoms are overtaken by the advancing interface and incorporated into the solid.

The details of the engulfment process may vary. In this paper the two cases of stepwise growth and continuous growth are treated. (We are not concerned here with predicting ${ }^{6.7}$ the transition from the former to the latter as the driving force grows.) The engulfment equation is combined with an interface velocity equation and steady-state solutions to the one-dimensional diffusion and heat equations to form a system capable of predicting crystallization behavior.

\section{SOLUTE TRAPPING}

The usual picture ${ }^{8}$ of interface advance consists of solvent and solute atoms in the liquid independently jumping across the interface and joining the solid structure. The growth rate is found by summing the net jumping rates for each species. It now seems, however, that the incorporation of an atom into the crystal is a process which occurs much more easily and much more rapidly than the solute-solvent exchange process, which is diffusive. ${ }^{4}$ We expect that the crystallization process is not a difficult one in metallic melts. There is evidence ${ }^{5}$ that the rate of the interface mechanism for growth is limited only by the frequency of impingement of atoms upon the interface. This means that the energy barrier which an atom must surmount in order to join the crystal is insignificant. In addition, such a jump is not a large, diffusive-like jump of length $\lambda$. The rearrangement step, drawn schematically in Fig. 1, is a shift from an equilibrium position in the liquid structure to that in the solid structure, and is of much smaller distance.

Figure 1 illustrates a heuristic model of solute trapping in two dimensions. Each atom in the solid and in the liquid is vibrating about is equilibrium position created by the shell of its nearest neighbors. The numbered atoms constitute the monolayer in the liquid immediately to the right of the interface. The lone impurity or $B$ atom is number 4 in this monolayer. The equilibrium segregation coefficient is small because the lowest-energy arrangement of A-B bonds is with the A-B-A bond angle at $60^{\circ}$ and the $\mathrm{B}$ atom is more likely to

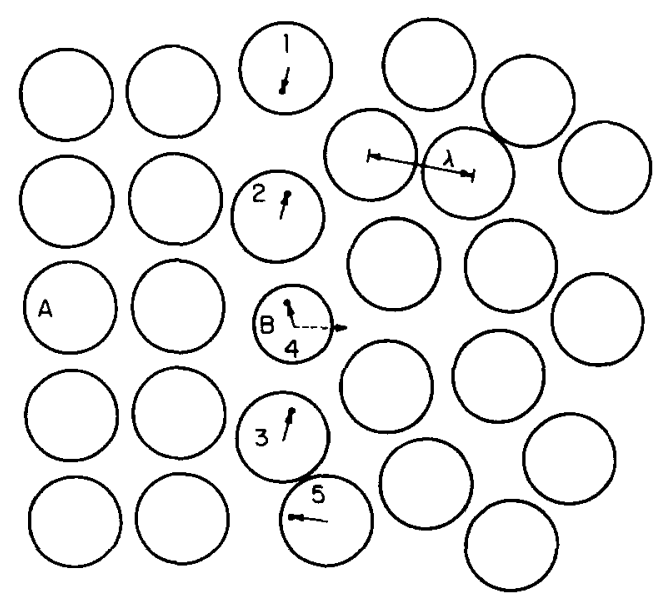

FIG. 1. Choreography of solute trapping. find such a configuration in the liquid. Now suppose that the crystal grows a distance $\lambda$ by the indicated motions of atoms $1,2,3$, and 5 . Atoms 4 finds the potential well in which it vibrates shifting toward the lattice position and gaining potential energy. As indicated by the solid arrow, the atom is dragged along by its potential well, unless it happens to be adjacent to a configuration in the liquid which allows it to "roll" down the right side of the potential hill which it is being dragged up. We will assume the fraction, $\alpha$, of impurity atoms moving to the lattice deposition to be unity for the rest of our calculations. ${ }^{9}$

If growth occurs by the rapid lateral passage of steps of height $\lambda$, then the average time between the passage of steps is $\tau=\lambda / u$, where $u$ is the growth velocity. The time it takes atoms 2,3 , and 4 to shift to their lattice positions is negligible when compared to $\tau$, and the shifts can be treated as virtually instantaneous. The impurity then tries to diffuse back to the liquid. If it does not do so before a time $\tau$ expires, then the interface advances across another monolayer and the impurity is permanently buried into the bulk of the solid, where diffusion is negligible.

If continuous solidification occurs, the picture is not so clear. The impurity atom is being dragged toward its lattice site over a time of order $\tau$. During this same time, it is trying to diffuse away.

The model presented here will not predict whether stepwise growth or continuous growth will occur, but the results depend upon the answer to this question. References 6 and 7 indicate that for sufficiently small driving forces the step mechanism will operate and for sufficiently large driving forces the interface will advance continuously. The critical driving force, where the transition occurs, will be very large if the interface is discrete. It is still open to question whether

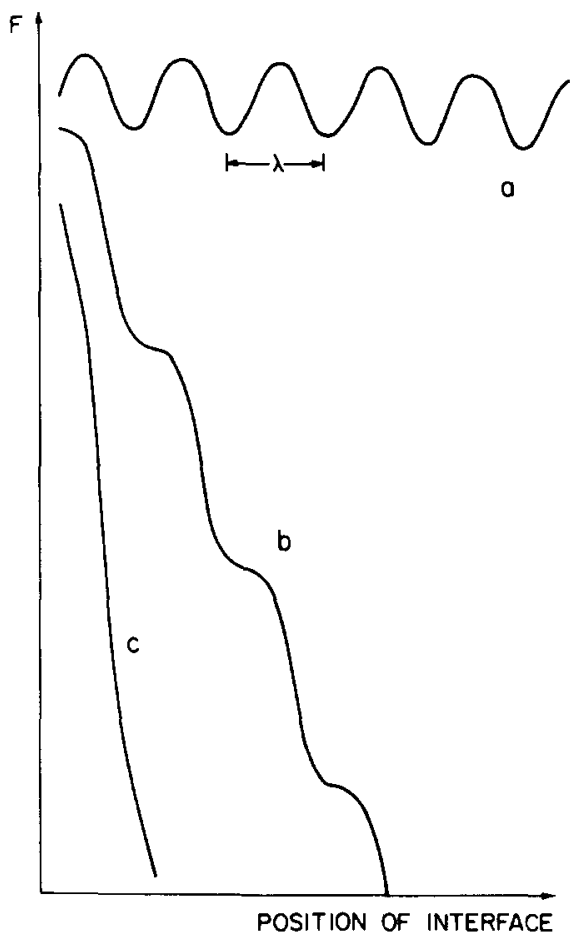

FIG. 2. Free energy of system as a function of interface position. (a) Small driving force, (b) critical driving force, and (c) large driving force. 
a driving force of such magnitude is attained in laser annealing of silicon. Figure 2, which is adapted from Ref. 6, is a diagram of the free energy of the system as the interface advances. In curve (a) the troughs represent complete filling of the surface monolayer of the crystal. The humps represent partial filling; some of the atoms in the monolayer have not yet shifted from their liquid positions to their lattice positions. In effect, the surface energy is a periodic function of position, with repeat distance $\lambda$. The surface energy is added to the driving force, expressed as a free energy gradient, to produce the curves in Fig. 2. The interface cannot be thermally activated across the humps in curve (a) because all of the atoms in the liquid monolayer adjacent to the interface must participate in such a jump; its only means of advancement is via the step mechanism. In curve (b), the driving force is just large enough for the interface to slide down the energy hill without having to climb. Note that the interface might be expected to move rapidly across the steeper parts of the curve and move more slowly across the flatter parts of the curve. Consequently, the impurity atom in Fig. 1 would experience a situation similar to that in stepwise growth: the interface moves rapidly across the monolayer and then pauses while the impurity tries to diffuse out. In curve (c), the driving force is so large that the humps are insignificant. In this limit, the interface advances steadily while the impurity tries to keep up with it.

\section{A. Stepwise growth}

In this regime, the interface can be considered locally planar (the wide range of stability of a planar interface is

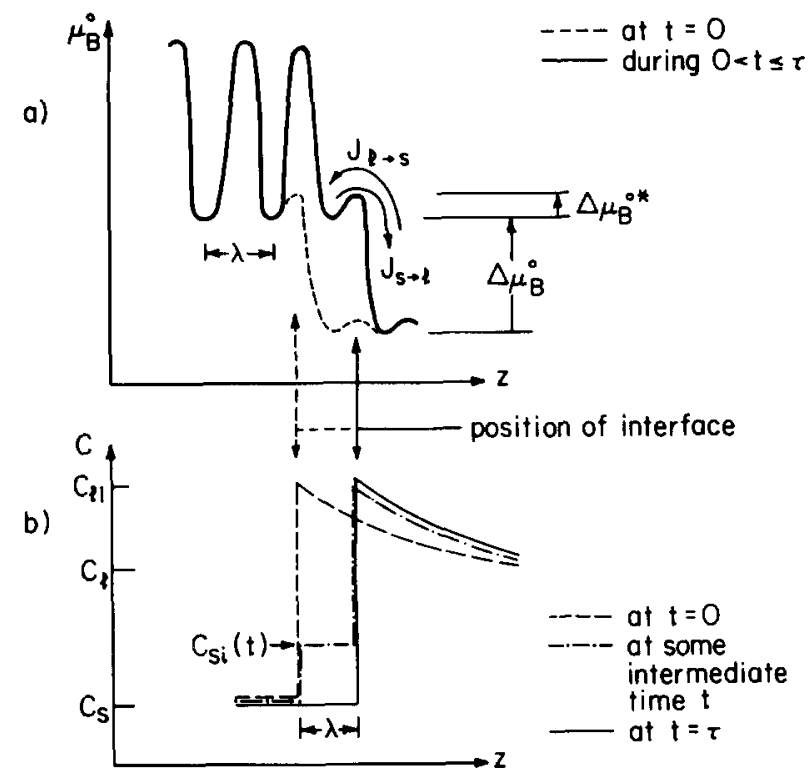

FIG. 3. Standard free energy of impurity atoms, concentration of impurity, and interface positon. (a) standard free energy of impurity at $t=0$ (dashed curve) and at $t=\tau$ (solid curve). For continuous growth, the curve evolves from the former to the latter in some unknown manner during $0<t<\tau$. For stepwise growth, the dashed curve instantaneously shifts to the solid curve at $t=0^{+}$and remains there for $0<t \leqslant \tau$. The solute fluxes $J_{s, 1}$ and $J_{1.5}$ across the activated state are depicted. (b) solute concentration drawn as a continuous function of position at $t=0$ (dashed curve), at intermediate time (dot-dash curve), and at $t=\tau$ (solid curve). $C_{s i}(t)$ drops during stepwise growth from $C_{l i}$ at $t=0$ to $C_{s}$ at $t=\tau$. discussed in Ref. 10), as depicted in Fig. 3. The advance of the interface at $t=0$ incorporates one liquid monolayer, including all solute atoms, into the growing crystal. If $C_{s i}$ is the impurity concentration in the newly-created solid interfacial monolayer and $C_{l i}$ is the impurity concentration in the liquid at the interface (to be determined by the solution to the diffusion equation), then $C_{s i}(t=0)=C_{l i}$. The impurity atoms in the solid monolayer then have some time to diffuse back into their low-energy states in the liquid. Those remaining are frozen into the bulk of the solid as the next monolayer is added at $t=\tau$, whereupon the entire process repeats itself. The negligible diffusivity in the bulk of the solid results in the composition of the growing solid,, $C_{s}$, being equal to that in the monolayer at the instant it is covered: $C_{s}=C_{s i}(\tau)$.

Chemical rate theory gives us expressions for the diffusive impurity flux across the inteface for $0<t<\tau$. The flux into the liquid from the solid monolayer is given by

$$
J_{s \rightarrow I}(t)=f_{s} \nu_{s} \lambda \gamma_{s} C_{s i}(t) \exp \left(\frac{-\Delta \mu_{\mathrm{B}}^{0 *}}{R T_{i}}\right),
$$

where $f_{s}$ is the fraction of sites in the solid monolayer where an atomic jump can occur, $v_{s}$ is the attempt frequency, which is on the order of an atomic vibrational frequency, $\gamma$, is the activity coefficient of solute in the solid, $C_{s i}$ is the instantaneous impurity concentration in the surface layer of the solid, $\Delta \mu_{\mathrm{B}}^{0 *}$ is the molar free energy of activation to the transition state, $R$ is the gas constant, and $T_{i}$ is the interface temperature. Similarly, the diffusive flux in the opposite direction is

$$
J_{l \rightarrow s}=f_{l} v_{l} \lambda \gamma_{l} C_{l i} \exp \left(\frac{-\Delta \mu_{\mathrm{B}}^{0 *}-\Delta \mu_{\mathrm{B}}^{0}}{R T_{i}}\right),
$$

where $f_{l}$ is the fraction of sites in the liquid monolayer where such an atomic jump can occur, $v_{l}$ is the attempt frequency in the liquid, $\gamma_{1}$ is the activity coefficient of solute in the liquid, $C_{l i}$ is the solute concentration in the liquid at the interface, " and $\Delta \mu_{\mathrm{B}}^{0}$ is the change in the standard molar free energy of solute upon crystallization. Assuming negligible diffusivity in the bulk of the solid, mass conservation requires $\lambda d C_{s i} / d t=J_{l \rightarrow s}-J_{s \rightarrow l}$. Inserting Eqs. (1) and (2) into this, and defining the "interface interdiffusivity" $D_{i} \equiv \lambda^{2} f_{l} \nu_{l} \gamma_{s} \exp \left(-\Delta \mu_{\mathrm{B}}^{0 *} / R T_{i}\right)$ and the equilibrium segregation coefficient (at any undercooling)

$$
k_{e}\left(T_{i}\right) \equiv \frac{\gamma_{l}}{\gamma_{s}} \exp \left(-\Delta \mu_{\mathrm{B}}^{0} / R T_{i}\right)
$$

we obtain

$$
\frac{d C_{s i}}{d t}=\frac{D_{i}}{\lambda^{2}}\left[k_{e} C_{l i}-\frac{f_{s} v_{s}}{f_{l} v_{l}} C_{s i}(t)\right] .
$$

Detailed balance at equilibrium requires $f_{s} v_{s}=f_{l} v_{l}$. Equation (3) becomes

$$
\frac{d C_{s i}}{d t}=\frac{-D_{i}}{\lambda^{2}}\left[C_{s i}(t)-k_{e} C_{l i}\right],
$$

a differential equation for $C_{s i}(t)$ with the boundary condition $C_{s i}(0)=C_{l i}$. The solution is

$C_{s i}(t)=C_{l i}\left\{k_{e}\left(T_{i}\right)+\left[1-k_{e}\left(T_{i}\right)\right] \exp \left[\frac{-D_{i} t}{\lambda^{2}}\right]\right\}$. 
Diffusion to and from this layer comes to a halt when the next monolayer is added at $t=\tau=\lambda / u$, and the remaining solute is permanently frozen into the solid. Consequently, if $X_{s}$ is the mole fraction of impurity in the solid and $X_{l i}$ is the mole fraction of impurity in the liquid at the interface, and if we ignore the volume change upon crystallization,

$$
\begin{aligned}
& X_{s}\left(X_{l i}, u, T_{i}\right) \\
& \quad=X_{l i}\left\{k_{e}\left(T_{i}\right)+\left[1-k_{e}\left(T_{i}\right)\right] \exp \left[\frac{-D_{i}}{u \lambda}\right]\right\} .
\end{aligned}
$$

We expect, ${ }^{12}$ for a sharp interface, $D_{i} \sim D_{L}$. Preliminary plots of Eq. (4) are made using $T_{i}=T_{M}$ and $D_{i}=D_{L}$ in Figs. 4 and 5 . Solutions for $T_{i}(u)$ for several systems of interest are in progress. Note that the model predicts equilibrium segregation for $u<D_{i} / \lambda$ and complete solute trapping for $u>D_{i} / \lambda$, with the transition occurring over approximately an order of magnitude in $u$. The location of the transition will depend upon $T_{i}(u)$ and $D_{i}$. However, the limits will remain unchanged as long as the liquid is so dilute that there is not interference from the thermodynamic limits to trapping discussed by Baker and $\mathrm{Cahn}^{13}$ and by Cahn, Coriell, and Boettinger. ${ }^{10}$

\section{B. Continuous growth}

When the interface advances at a relatively uniform velocity, the potential surrounding the solute atom as it moves during regrowth is constantly changing in an unknown way (see Fig. 3). Wood ${ }^{14}$ has suggested a molecular dynamics calculation to explore this behavior. It is possible, however, without such detailed knowledge, to write solutions for dif-

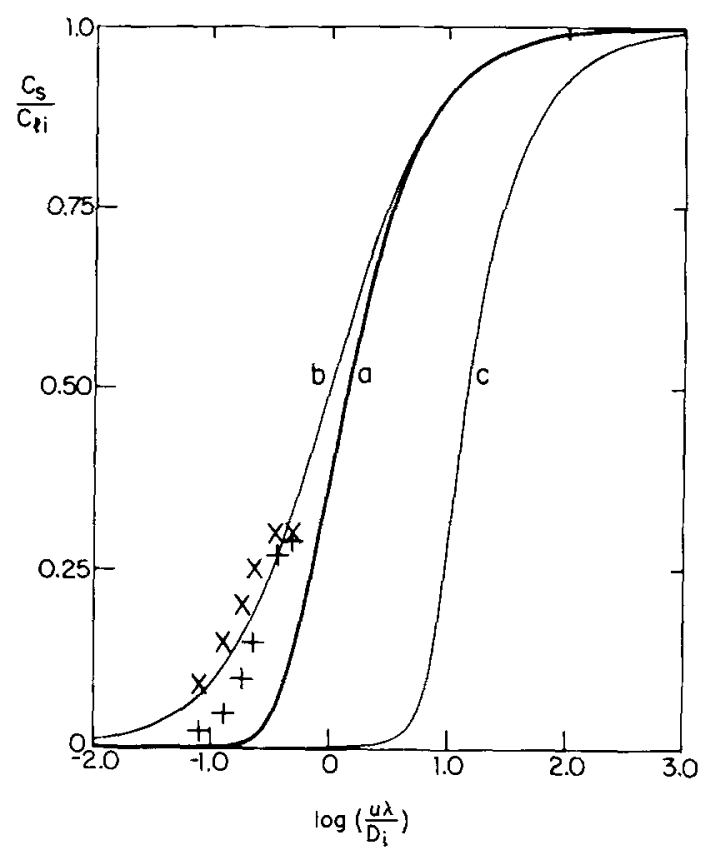

FIG. 4. Bismuth $\left(k_{\mathrm{e}}=7 \times 10^{-4}\right)$ segregation in silicon. (a) stepwise growth model (this work), (b) continuous growth model (this work) and JGL, and (c) Baker model. X - Baeri et al. data $\langle 111\rangle$. + -Poate data $\langle 100\rangle$. Data plotted assuming $D_{t} / \lambda=D_{L} / \lambda=10 \mathrm{~m} / \mathrm{s}$. Theoretical curves plotted assuming $\alpha=1, T_{i}=T_{M}$.

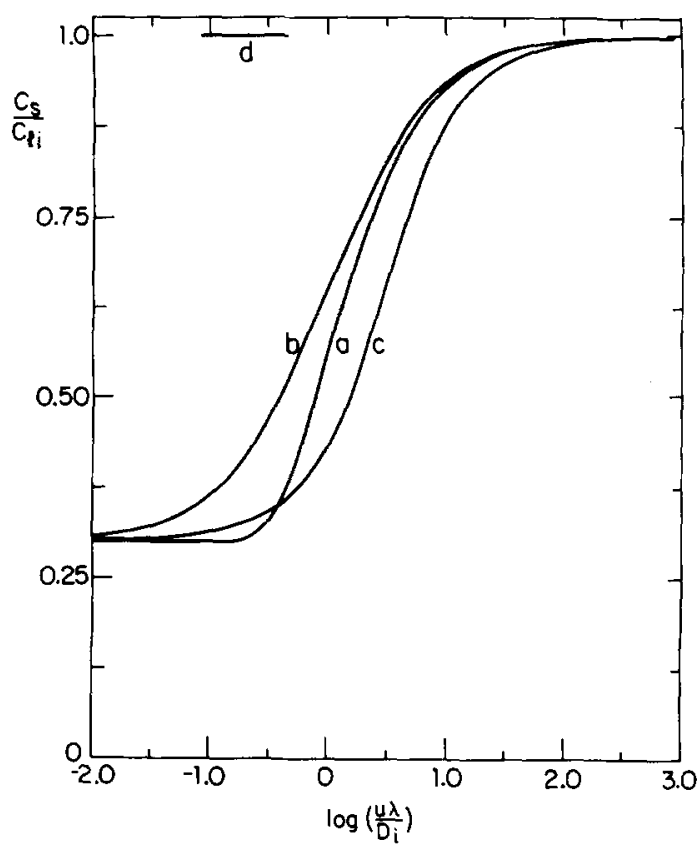

FIG. 5. Arsenic $\left(k_{e}=0.3\right)$ segregation in silicon: (a) stepwise growth model (this work); (b) continuous growth model (this work) and JGL; (c) Baker model. (d) Data, plotted assuming $D_{i} / \lambda=D_{L} / \lambda=10 \mathrm{~m} / \mathrm{s}$. Theoretical curves plotted assuming $\alpha=1, T_{i}=T_{M}$.

fusive-type rate equations describing solute jumping across the interface, if we assume that we have steady-state fluxes. We also assume that some kind of an activated transition state must be reached in order for any jump to occur, as shown in Fig. 3. Once again, the activation energy for solutesolvent exchange (jumps of length $\lambda$ ) must be significantly greater than that for crystallization (jumps of length $<\lambda$ ). Without linearizing (which, we will see, leads to the standard diffusion equation and Baker's results ${ }^{10}$ ) the resulting rate equations for solute jumps of length $\lambda$ across a sharp interface, we find the steady-state solution in a reference frame moving with velocity $u$, its origin fixed on the interface.

In such a reference frame, the requirement of the steady state in one dimension is that the flux be the same everywhere so that no solute buildup occur with time. If $J(z)$ is the net diffusive impurity flux and $C(z)$ the impurity concentration, the steady-state requirement is

$$
\frac{\partial}{\partial z}(J-u C)=0
$$

because $u C(z)$ is the nondiffusive flux due to center-of-mass motion. Since $\partial C / \partial z=\left(C_{l i}-C_{s i}\right) / \lambda$ and $^{15}$

$\partial J / \partial z=\left(J_{s \rightarrow l}-J_{l \rightarrow s}\right) / \lambda$, the steady-state requirement becomes

$$
J_{s \rightarrow l}-J_{l \rightarrow s}=u\left(C_{l i}-C_{s i}\right) .
$$

The rate Eqs. (1) and (2) from the previous section are inserted into Eq. (5) to give us

$$
\begin{aligned}
u\left(C_{l i}\right. & \left.-C_{s i}\right) \\
& =f_{l} v_{l} \lambda \exp \left(\frac{-\Delta \mu_{\mathrm{B}}^{\mathrm{O} *}}{R T_{i}}\right)\left(C_{s} \gamma_{s}-C_{l i} \gamma_{l} \exp \frac{-\Delta \mu_{\mathrm{B}}^{\mathrm{o}}}{R T_{i}}\right) .
\end{aligned}
$$

With $D_{i}$ and $k_{e}\left(T_{i}\right)$ defined as before, and defining the dimensionless velocity $\beta \equiv u \lambda / D_{i}$, this becomes 


$$
\beta\left(C_{l i}-C_{s i}\right)=C_{s i}-k_{e} C_{l i},
$$

or, with negligible diffusion in the solid so that $C_{s}=C_{s i}$ (which, in a steady-state formulation, does not vary with time), we have

$$
k^{\prime}=\frac{C_{s}}{C_{l i}}=\frac{\beta+k_{e}}{\beta+1} .
$$

This expression ${ }^{16}$ approaches the limiting values of $k_{e}$ when $u \rightarrow 0$ and 1 when $u>D_{i} / \lambda$. The transition from equilibrium segregation to complete trapping, shown in Figs. 4 and 5, begins at lower velocities than it does in stepwise growth, but ends at the same velocities. These two cases may represent limits for solute trapping during the transition regime where interface motion is neither virtually instantaneous and stepwise nor virtually constant and continuous.

\section{Growth velocity}

For pure systems, the interface velocity has been written ${ }^{17-19}$

$$
u=f k_{i} \lambda\left[1-\exp \left(\Delta \bar{G} / R T_{i}\right)\right],
$$

where $f(h k l)$ is the fraction of interface sites which are growth sites, $k_{i}$ is the atomic hopping frequency across the interface, and $\Delta \bar{G}$ is the change in molar free energy upon crystallization. In metallic melts, it has been proposed ${ }^{15,20-22}$ that growth is impingement limited, resulting in $k_{i} \approx v_{l} \approx u_{s} / \lambda$ and

$$
u=f u_{s}\left[1-\exp \left(\Delta \bar{G} / R T_{i}\right)\right],
$$

where $u_{s}$ is the speed of sound. In alloy solidification, it seems difficult to treat the two species individually since we know they cannot be thought of as independent. Consequently, we will assume complete dependence, i.e., that the impurity atoms make the same jump as do the solvent atoms, that Eq. (7) still holds, and that $\Delta \bar{G}$ is given by the weighted average of the changes in the chemical potentials:

$$
\begin{aligned}
\Delta \bar{G} & =\left[\mu_{s}^{\mathrm{A}}\left(X_{s}\right)-\mu_{l}^{\mathrm{A}}\left(X_{l i}\right)\right]\left(1-X_{s}\right) \\
& +\left[\mu_{s}^{\mathrm{B}}\left(X_{s}\right)-\mu_{l}^{\mathrm{B}}\left(X_{l i}\right)\right]\left(X_{s}\right) .
\end{aligned}
$$

When we insert (7) into (8) the result is

$$
\begin{aligned}
& {\left[\mu_{l}^{\mathrm{A}}\left(X_{l i}\right)-\mu_{s}^{\mathrm{A}}\left(X_{s}\right)\right]\left(1-X_{s}\right)-\left[\mu_{s}^{\mathrm{B}}\left(X_{s}\right)-\mu_{l}^{\mathrm{B}}\left(X_{l i}\right)\right]} \\
& +R T_{i} \ln \left(1-u / f u_{s}\right)=0 .
\end{aligned}
$$

The first term is the decrease in free energy of the solvent during solidification; the second is the gain in free energy of the trapped solute. The final term is the free energy dissipated in driving the interface motion. Thompson and Spaepen ${ }^{23}$ have developed a reliable method of estimating the chemical potentials of interest here.

Note the approximation implicit in Eq. (8). The instantaneous freezing of a monolayer is accompanied by a change in free energy which is strictly given by

$\Delta \bar{G}_{s}=\left[\mu_{s}^{\mathrm{A}}\left(X_{l i}\right)-\mu_{l}^{\mathrm{A}}\left(X_{l i}\right)\right]\left(1-X_{l i}\right)+\left[\mu_{s}^{\mathrm{B}}\left(X_{l i}\right)-\mu_{l}^{\mathrm{B}}\left(X_{l i}\right)\right]\left(X_{l i}\right)$.

We see that such a process is only possible below the $T_{0}$ line on the phase diagram, since $T_{i} \leqslant T_{0}\left(X_{l i}\right)$, if and only if $\Delta \bar{G}_{s}$ $<0$. If $X_{i i}$ becomes too large, then $T_{0}$ will drop below $T_{i}$, and we will find the instantaneous freezing process unable to proceed. In this case, the passing ledge must wait for diffusion to eliminate enough solute from the liquid interfacial monolayer to bring $T_{0}\left(X_{l i}\right)$ back above $T_{i} .{ }^{24}$ We do not know how far beyond $T_{i}$ the system brings $T_{0}\left(X_{l i}\right)$ before the monolayer actually freezes; it is believed ${ }^{5,25}$ not to be very far. We avoid these difficulties by writing Eq. (8), which gives $\Delta \bar{G} \leqslant 0$ whenever solidification can occur. ${ }^{26}$ In addition, whenever $u$ is slowed enough to allow significant diffusive solute escape before the monolayer freezes, Eqs. (4) and (6) will predict significant diffusive solute escape, and the resulting error when $T_{i}>T_{0}$ may not be very large. Below the $T_{0}$ line, Eq. (8) actually gives the sum of the free energy dissipated to drive the interface motion and that dissipated to drive solute-solvent exchange. We are overestimating the former by assuming the latter to be insignificant ${ }^{27}$ when we combine Eq. (7) with Eq. (8). The resulting error goes to zero as the velocity increases and the solute experiences complete trapping. In any system, we will always have $T_{i}<T_{0}$ when the solution is sufficiently dilute. In summary, the approximation implicit in Eq. (8) is that the free energy dissipated in driving solutesolvent exchange is significantly less than the free energy dissipated in driving the interface motion.

Equations (4) and (9) or (6) and (9), are the two "response functions" of the interface to the local conditions. They are similar in some ways to other recently published models; comparisons are made in the next section.

\section{COMPARISON TO OTHER MODELS}

Baker model. This analysis ${ }^{10}$ treats a planar interface as a continuum. The energy of a solute atom $E(z)$ is a constant $E_{l}$ in the liquid, a constant $E_{s}$ in the solid, and is given the value $E_{i}$ at the center of the interface, which has width $2 \delta$. $E(z)$ varies linearly from $E_{l}$ to $E_{i}$ to $E_{s}$ across the interface. The standard diffusion equation is set up and solved in steady state, in a reference frame fixed on the interface, whose velocity is $u$. The ratio of the steady-state compositions on the two sides of the interface is the segregation coefficient. Baker's model does not predict the growth velocity.

In the moving reference frame, the diffusion equation is

$\partial C / \partial t=-u \nabla C+\nabla \cdot J$,

where $C(z)$ is the impurity concentration and $J(z)$ is the diffusive impurity flux. The approximation is made that the diffusive flux is proportional to the driving force,

$J=(D C / R T) \nabla \mu$, although the chemical potential gradient may be quite large. The chemical potential is written

$\mu(z)=E(z)+R T \ln C(z)$, giving

$$
J=-D \nabla C-(D C / R T) \nabla E .
$$

The first term is the familiar diffusive flux through a uniform medium. The second term represents solute sliding down an energy "hill" which is the interface. If $E_{i}=E_{s}$ and $\delta=\lambda$, the result is

$$
k^{\prime}=\frac{C_{s i}}{C_{l i}}=\frac{\beta+\ln k_{e}}{\beta+\frac{1}{k_{e}} \ln k_{e} \exp (-\beta)},
$$

where $\beta \equiv \lambda u / D_{i}$. This expression, plotted as curve (c) in Figs. 4 and 5 , goes to the same limits of equilibrium segregation and complete solute trapping as do ours, but the transi- 
tion occurs for small $k_{e}$ at velocities which are quite high compared to the diffusive speed $D_{L} / \lambda$. This is a direct consequence of the linear approximation which leads to Eq. (10). As $k_{e}$ becomes smaller, $E_{s}-E_{l}$ and hence $\nabla E$ become arbitrarily large. According to Eq. (10), an arbitrarily large diffusive flux is possible across the interface, which seems unlikely. It allows solute to escape back into the liquid when it might otherwise be trapped, if the flux were limited.

In our model for continuous growth at a sharp interface, we would arrive at a similar result if we were to linearize $J=J_{s \rightarrow l}-J_{l \rightarrow s}$ in $\nabla \mu$. By refraining from doing so, we find the diffusive flux approaching an upper limit when $\nabla \mu$ becomes so large (i.e., $k_{e}$ becomes so small) that the back reaction $J_{l \rightarrow s}$ goes to zero.

Jackson-Gilmer-Leamy Model. This analysis, ${ }^{28}$ which will be referred to as JGL, sets up rate equations for hopping of both solute and solvent atoms across the interface. These equations are used to describe both crystallization and solute-solvent redistribution, ignoring the basic difference between the two atomic processes. The growth velocity is taken to be the sum of the net fluxes of A and B atoms, and an expression for the solid composition is arrived at by taking the ratio of the net fluxes. In addition, it is supposed that a fraction, $\alpha$, of the impurity atoms are trapped in some manner into the growing crystal. To the impurity crystallization flux, determined by one of the rate equations, they add this trapping flux.

The rate equations for solute hopping are written in the same form as those for solvent hopping. JGL's equations (1) become our equations (1), (2),

$$
\begin{aligned}
& J_{l \rightarrow s}^{\mathrm{A}}=C_{l i}^{\mathrm{A}} f_{l}^{\mathrm{A}} v_{l}^{\mathrm{A}} \lambda \exp \left(-\Delta \mu_{\mathrm{A}}^{\mathrm{O}} / R T_{i}\right), \\
& J_{s \rightarrow 1}^{\mathrm{A}}=C_{s}^{\mathrm{A}} f_{s}^{\mathrm{A}} v_{s}^{\mathrm{A}} \lambda \exp \left[\left(\Delta \mu_{\mathrm{A}}^{0}-\Delta \mu_{\mathrm{A}}^{\mathrm{O} *}\right) / R T_{i}\right],
\end{aligned}
$$

and $J_{\text {trap }}^{\mathrm{B}}=\alpha u C_{l i}^{\mathrm{B}}$. The notation of JGL translates into ours as follows: $R_{\mathrm{A}}^{+}=\Omega J_{l \rightarrow s}^{\mathrm{A}}, R_{\mathrm{A}}^{-}=\Omega J_{s \rightarrow l}^{\mathrm{A}}, R_{\mathrm{B}}^{+}=\Omega J_{l \rightarrow s}^{\mathrm{B}}$, $R_{\mathrm{B}}^{-}=\Omega J_{s \rightarrow 1}^{\mathrm{B}}, Q_{\mathrm{A}}=\Delta h_{\mathrm{A}}^{0 *}, Q_{\mathrm{B}}=\Delta h_{\mathrm{B}}^{0 *}-R T \ln \gamma_{s}$, $E=\Delta h_{\mathrm{B}}^{0}+R T \ln \gamma_{s} / \gamma_{1}$,

$$
\begin{aligned}
& \left(R_{\mathrm{A}}^{+}\right)_{0}=\lambda f_{l}^{\mathrm{A}} v_{l}^{\mathrm{A}} \exp \left(\Delta s_{\mathrm{A}}^{0 *} / R\right), \\
& \left(R_{\mathrm{B}}^{+}\right)_{0}=\lambda f_{l}^{\mathrm{B}} v_{l}^{\mathrm{B}} \exp \left[\left(\Delta s_{\mathrm{B}}^{0 *}+\Delta s_{\mathrm{B}}^{0}\right) / R\right], \\
& \left(R_{\mathrm{A}}^{-}\right)_{0}=\lambda f_{s}^{\mathrm{A}} v_{s}^{\mathrm{A}} \exp \left[\left(\Delta s_{\mathrm{A}}^{0 *}+\Delta s_{\mathrm{A}}^{\mathrm{f}}\right) / R\right],
\end{aligned}
$$

and $\left(R_{\mathrm{B}}^{-}\right)_{0}=\lambda f_{s}^{\mathrm{B}} v_{s}^{\mathrm{B}} \exp \left(\Delta s_{\mathrm{B}}^{0 *} / R\right)$,

where $h_{\mathrm{A}, \mathrm{B}}^{0}=\mu_{\mathrm{A}, \mathrm{B}}^{0}+T s_{\mathrm{A}, \mathrm{B}}^{0}$ is the standard partial molar enthalpy of $A$ and $B$, and $\Omega$ is the molar volume. (The previously implicit superscript $B$ is written explicitly only in this section.) The standard states are defined to be pure $\mathbf{A}$ and pure B. Detailed balance is invoked for both species in equilibrium at temperature $T_{e}$. Applied to the solvent, it requires $f_{l}^{\mathrm{A}} \nu_{l}^{\mathrm{A}} / f_{s}^{\mathrm{A}} \gamma_{s}^{\mathrm{A}}=\left(C_{s}^{\mathrm{A}} / C_{l i}^{\mathrm{A}}\right) \exp \left(\Delta \mu_{\mathrm{A}}^{0} / R T_{e}\right)$. For regular solutions in the solid and in the liquid at the interface, the requirement becomes JGL's Eq. (2): $f_{l}^{\mathrm{A}} v_{l}^{\mathrm{A}}=f_{s}^{\mathrm{A}} v_{s}^{\mathrm{A}}$. Recall from Sec. II that detailed balance for the minor component implies $f_{s}^{\mathrm{B}} v_{s}^{\mathrm{B}}=f_{l}^{\mathrm{B}} v_{l}^{\mathrm{B}}$, which gives, in the notation of JGL,

$$
R_{\mathrm{B}}^{-}=\frac{X_{s}^{\mathrm{B}}}{k_{e}}\left(R_{\mathrm{B}}^{+}\right)_{0} \exp \left(\frac{-E}{R T_{L}\left(X_{l i}^{\mathrm{B}}\right)}-\frac{Q_{\mathrm{B}}}{R T_{i}}\right)
$$

Here $T_{L}\left(X_{l i}^{\mathrm{B}}\right)$ is the liquidus temperature at composition $X_{l i}^{\mathrm{B}}$, which becomes $T_{M}^{\mathrm{A}}$ for sufficiently dilute solutions. Equation (13) is equivalent to JGL's Eq. (4). When pure B does not have the same equilibrium crystal structure as does pure A, it should be noted that the latent heat needed for such a calculation is that of pure B when B is in the metastable crystal structure which is crystallizing out of the liquid in the binary solidification problem: it is not the latent heat of fusion of pure $B$ liquid to the pure $B$ equilibrium solid phase. It may be possible in such cases to estimate $\Delta h_{\mathrm{B}}^{0}$ from a fit of the relevant portion of the phase diagram to a regular solution model.

The growth velocity is given by the sum of the net fluxes,

$$
u=\Omega\left(J_{l \rightarrow \mathrm{s}}^{\mathrm{A}}-J_{s \rightarrow l}^{\mathrm{A}}+J_{l \rightarrow s}^{\mathrm{B}}+J_{\text {trap }}^{\mathrm{B}}-J_{s \rightarrow l}^{\mathrm{B}}\right),
$$

and the solid composition is deduced from the ratio of the net fluxes:

$$
\frac{C_{s}^{\mathrm{B}}}{C_{s}^{\mathrm{A}}}=\frac{J_{l \rightarrow s}^{\mathrm{B}}+J_{\mathrm{trap}}^{\mathrm{B}}-J_{s \rightarrow l}^{\mathrm{B}}}{J_{l \rightarrow s}^{\mathrm{A}}-J_{s \rightarrow l}^{\mathrm{A}}},
$$

These are combined to produce JGL's Eq. (10):

$$
k^{\prime} \equiv \frac{C_{s}^{\mathrm{B}}}{C_{l i}^{\mathrm{B}}}=\frac{\left(J_{l \rightarrow s}^{\mathrm{B}} / C_{l i}^{\mathrm{B}}\right)+\alpha u}{\left(J_{s \rightarrow 1}^{\mathrm{B}} / C_{s}^{\mathrm{B}}\right)+u} .
$$

In terms of JGL's dimensionless velocity $V \equiv u C_{l i}^{\mathrm{A}} / J_{l \rightarrow s}^{\mathrm{A}}$, we have

$$
k^{\prime}=\frac{\alpha V+\left(C_{l i}^{\mathrm{A}} J_{l \rightarrow s}^{\mathrm{B}} / C_{l i}^{\mathrm{B}} J_{l \rightarrow s}^{\mathrm{A}}\right)}{V+\left(C_{l i}^{\mathrm{A}} J_{s \rightarrow l}^{\mathrm{B}} / C_{s}^{\mathrm{B}} J_{l \rightarrow s}^{\mathrm{A}}\right)} .
$$

We get the ratios of the fluxes from the rate equations:

$$
\frac{J_{l \rightarrow s}^{\mathrm{B}}}{J_{l \rightarrow s}^{\mathrm{A}}}=\frac{C_{l i}^{\mathrm{B}} f_{l}^{\mathrm{B}} v_{l}^{\mathrm{B}} \gamma_{s}}{C_{l i}^{\mathrm{A}} f_{l}^{\mathrm{A}} v_{l}^{\mathrm{A}}} k_{e} \exp \left[\left(\Delta \mu_{\mathrm{A}}^{0 *}-\Delta \mu_{\mathrm{B}}^{\mathrm{O} *}\right) / R T_{i}\right]
$$

and

$$
\frac{J_{s \rightarrow l}^{\mathrm{B}}}{J_{l \rightarrow s}^{\mathrm{A}}}=\frac{C_{s}^{\mathrm{B}} \gamma_{s} f_{s}^{\mathrm{B}} v_{s}^{\mathrm{B}}}{C_{l i}^{\mathrm{A}} \gamma_{l} f_{l}^{\mathrm{A}} v_{l}^{\mathrm{A}}} \exp \left[\left(\mu_{\mathrm{A}}^{0 *}-\Delta \mu_{\mathrm{B}}^{0 *}\right) / R T_{i}\right] .
$$

JGL assume $f_{l}^{\mathrm{B}} v_{l}^{\mathrm{B}} \exp \left[\left(\Delta s_{\mathrm{B}}^{0 *}+\Delta s_{\mathrm{B}}^{0}\right) / R\right]$

$$
=f_{l}^{\mathrm{A}} v_{l}^{\mathrm{A}} \exp \left(\Delta s_{\mathrm{A}}^{0 *} / R\right) \text {, which turns Eq. (15) into }
$$

$$
k^{\prime}=\frac{\alpha V+\gamma_{s} k_{e} \exp \left(\frac{\Delta h_{\mathrm{A}}^{0 *}-\Delta h_{\mathrm{B}}^{0 *}}{R T_{i}}-\frac{\Delta s_{\mathrm{B}}^{0}}{R}\right)}{V+\gamma_{s} \exp \left(\frac{\Delta h_{\mathrm{A}}^{0 *}-\Delta h_{\mathrm{B}}^{0 *}}{R T_{i}}-\frac{\Delta s_{\mathrm{B}}^{0}}{R}\right)}
$$

this is equivalent to JGL's Eq. (15). If we assume instead that $f_{l}^{\mathrm{A}} v_{l}^{\mathrm{A}}=f_{l}^{\mathrm{B}} v_{l}^{\mathrm{B}} \equiv f v$, the result is

$$
k^{\prime}=\frac{V+\gamma_{s} k_{e} \exp \left(\frac{\Delta \mu_{\mathrm{A}}^{0 *}-\Delta \mu_{\mathrm{B}}^{0 *}}{R T_{i}}\right)}{V+\gamma_{s} \exp \left(\frac{\Delta \mu_{\mathrm{A}}^{0 *}-\Delta \mu_{\mathrm{B}}^{0 *}}{R T_{i}}\right)},
$$

if $\alpha=1$.

$$
\text { If we choose } \gamma_{\mathrm{B}}^{s} \exp \left(-\Delta \mu_{\mathrm{B}}^{0 *} / R T_{i}\right)
$$
$=\gamma_{\mathrm{A}}^{s} \exp \left(-\Delta \mu_{\mathrm{A}}^{0 *} / R T_{i}\right)$, parallel to the suggestion of $\mathrm{JGL}$ that $Q_{\mathrm{A}}=Q_{\mathrm{B}}$ for substitutional alloys, then we can show that $V$ becomes $u \lambda / D_{i}$. Equation (17) then becomes 

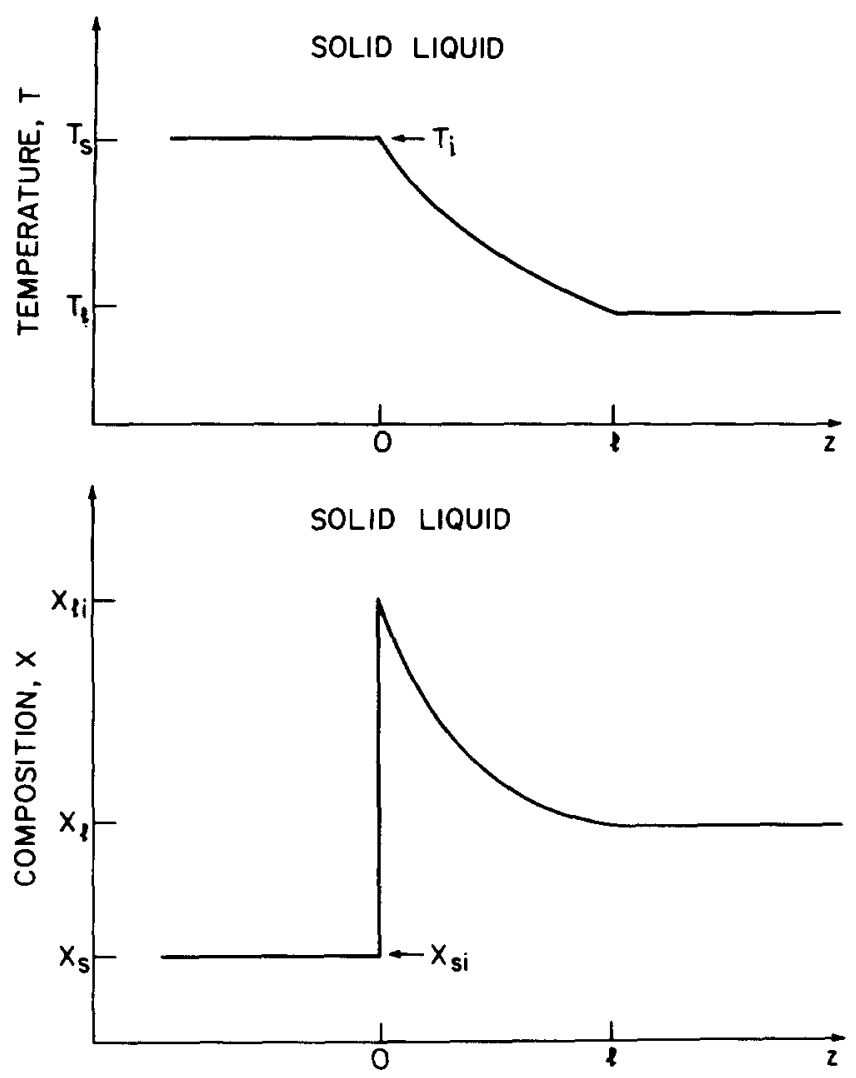

FIG. 6. Steady-state temperature and composition profiles in reference frame moving with interface.

$$
k^{\prime}=\left(\beta+k_{e}\right) /(\beta+1),
$$

which is formally identical to our continuous growth result, Eq. (6). However, we will see that in this case the growth velocity is limited by the diffusive speed; i.e., $\beta$ is restricted to the range $0 \leqslant \beta \leqslant 1$. From $\mathrm{Eq}$. (14), it follows that $u \approx\left(J_{l \rightarrow s}^{\mathrm{A}} / \mathrm{C}_{l i}^{\mathrm{A}}\right)\left[1-\exp \left(\Delta \mu_{\mathrm{A}}^{0} / R T_{i}\right)\right]$. But if $\Delta \mu_{\mathrm{A}}^{0 *}=\Delta \mu_{\mathrm{B}}^{0 *}$ $-R T \ln \gamma_{s}$, then from Eq. (12) we get $J_{l \rightarrow s}^{\mathrm{A}} / C_{l i}^{\mathrm{A}}=D_{i} / \lambda$, and hence $u \leqslant D_{i} / \lambda$. Such an upper limit on the growth velocity results in an effective kinetic limit to the segregation coefficient at

$$
k^{\prime}\left(u_{\max }\right)=\left(1+k_{e}\right) / 2 .
$$

This result is the left half of curve (b) in Figs. 4 and 5, in the region $\log \left(u \lambda / D_{i}\right) \leqslant 0$. We would arrive at a similarly truncated curve if, instead of Eq. (7), we had written ${ }^{29}$

$$
u=\frac{D_{i}}{\lambda}\left[1-\exp \left(\Delta \bar{G} / R T_{i}\right)\right]
$$

\section{DIFFUSION AND HEAT EQUATIONS}

We have seen how the interface responds to the local conditions of temperature and composition. In order to make any comparison with experiment, we must derive the local conditions from the ambient conditions. In general, solutions to the heat equation and the diffusion equation are quite complicated and require sophisticated computer techniques. ${ }^{30-32}$ A macroscopic steady-state analysis, however, is relatively simple. Consider a semi-infinite crystal growing with a constant velocity $u$ to the right into a semi-infinite liquid of bulk composition $X_{l}$ and temperature $T_{l}$. Figure 6 depicts the situation in a reference frame moving with respect to the center of mass, its origin fixed on the interface. The interface temperature is $T_{i}$; its composition is $X_{l i}$ on the liquid side and $X_{s i}$ on the solid side. The temperature gradient at the interface is $\nabla T_{l i}$ on the liquid side and $\nabla T_{s i}$ on the solid side. Convection in the liquid is assumed to effect complete mixing at a distance $l$ from the interface; between $z=0$ and $z=l$ is a liquid boundary layer, where transport is assumed to occur only by thermal and impurity diffusion. ${ }^{33}$ Ignoring the Soret effect and any dependence of the diffusivity $D_{L}$ upon concentration or temperature, Fick's law is written $\partial C / \partial t=D_{L} \nabla^{2} C+u \nabla C$ in a reference frame moving to the right with velocity $u$ with respect to the center of mass. In steady state, $\partial C / \partial t=0$ so $\nabla^{2} C=-u \nabla C / D_{L}$, or equivalently, $\nabla^{2} X=-u \nabla X / D_{L}$. In a similar manner we obtain $\nabla^{2} T=-u \nabla T / D_{T}$ for Fourier's law for thermal diffusion, where the thermal diffusivity in the liquid $D_{T}$ is given by $K / \rho C_{p}$. Here $K$ is the thermal conductivity, $\rho$ is the density, and $C_{p}$ is the specific heat at constant pressure. Note that these two equations require the sign of the curvature to be opposite to that of the gradient. If the temperature and composition are to remain bounded, the only possible steadystate profile in the solid $(z<0)$ is $T=$ constant, $C=$ constant. Figure 5 has been drawn accordingly: $X_{s}=X_{l i}$, $T_{s}=T_{i}, \nabla T_{s i}=0$. The other boundary conditions are

$$
-D_{L} \nabla X_{l i}=\left(X_{l i}-X_{s}\right) u
$$

(conserving solute at the interface) and

$$
-D_{T} \nabla T_{l i}=L\left(T_{i}, X_{s}, X_{l i}\right) u / C_{p},
$$

(conserving energy at the interface). We may be able to estimate the latent heat $L$ rejected upon forming solid of composition $X_{s}$ from liquid of composition $X_{l i}$ at temperature $T_{i}$ from thermodynamic data. The solutions are

$$
\begin{aligned}
& X_{l i}=X_{s}+\left(X_{l}-X_{s}\right) \exp \left(+u l / D_{L}\right), \\
& T_{i}=T_{l}+\left[1-\exp \left(-u l / D_{T}\right)\right] L / C_{p} .
\end{aligned}
$$

In principle, Eqs. (4 or 6), (9), (20), and (21) can be solved simultaneously to give $X_{s}, T_{i}, X_{l i}$, and $u$ in terms of $X_{l}$ and $T_{l}$. Numerical solutions for certain systems of interest are in progress.

\section{APPLICATION TO LASER ANNEALING}

The previous analysis can be applied to periods of steady-state behavior during processes which, in their entirety, are not steady. For example, Levi and Mehrabian ${ }^{34}$ find a transient period and a steady growth period in their analysis of the solidification of undercooled metallic liquid droplets. Regrowth from laser-induced melting also appears to occur at velocities changing by less than a factor or two during most of the regrowth. ${ }^{30,31}$ For the latter case, however, virtually all of the heat is extracted through the solid and Eq. (21) must be replaced.

\section{A. Modification of steady-state solution to heat equation}

When a temperature gradient exists in the solid at the interface, conservation of energy at the interface requires $\mathrm{Eq}$. 


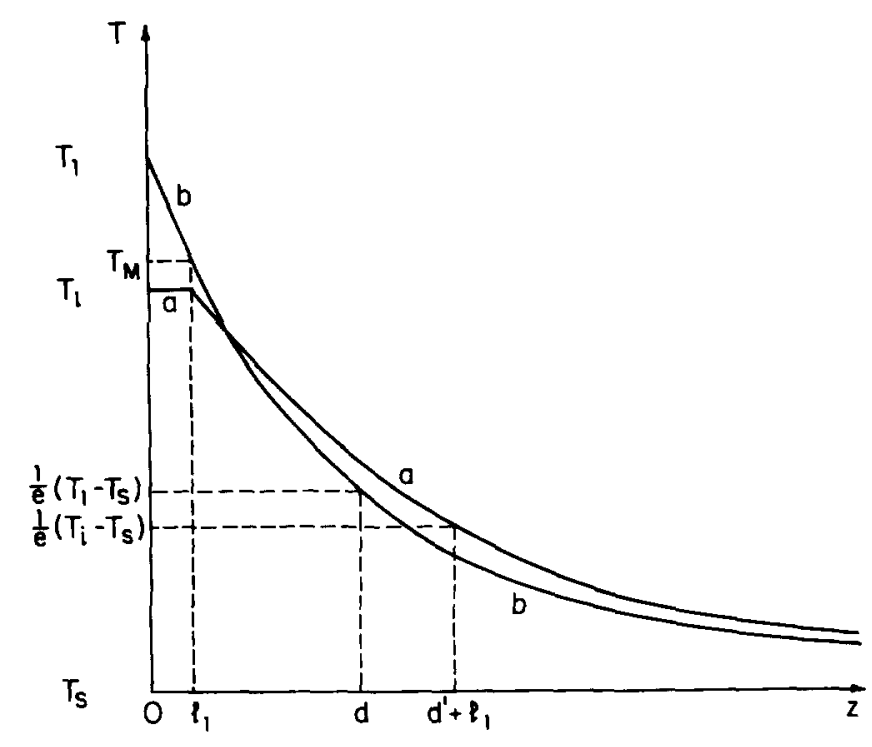

FIG. 7. Laser-induced temperature profiles in solid and liquid. (a) Immediately after laser pulse, and (b) at commencement of steady-state regrowth.

\section{(19) to be rewritten}

$$
D_{T}\left(\nabla T_{l i}-\nabla T_{s i}\right)=L u / C_{p} .
$$

For thermal transport during laser annealing, we use this to replace Eq. (21) as the fourth equation of the system to be solved. We can approximately determine the temperature gradients as follows.

For most cases of practical interest ${ }^{35}$ an incident planar laser pulse of duration $t_{p}$ creates a temperature profile whose decay length $d$ is given by $d=\left(2 D_{T} t_{p}\right)^{1 / 2}$, where $D_{T}$ is the thermal diffusivity of the solid; we will ignore the variation with temperature of $D_{T}$ and the difference between this and the thermal diffusivity of the liquid. The initial profile, in a semi-infinite solid occupying the half-space $z \geqslant 0$, is roughly exponential as shown in Fig. 7; the details of the deviations near the surface $(z=0)$ are unimportant. The pulse energy per unit area $E_{p}$ and the pulse duration dictate $l_{1}$, the initial depth of melting. We let the absorbed energy per unit area $E_{0}$ account for the latent heat of melting and for the temperature rise in the material. We assume that the exponential profile is not significantly disturbed by the presence of a solid-liquid interface ${ }^{36}$ during melting, and we locate the melt front by finding the point of the exponential temperature profile where $T(z)=T_{M} \cdot{ }^{37}$ Letting $T_{1}$ be the surface (i.e., at $z=0$ ) temperature, we have

$$
T(z)-T_{s}=\left(T_{1}-T_{s}\right) \exp (-z / d) .
$$

We locate the initial depth of melting at $z=l_{1}$ by setting

$$
T_{M}=T\left(l_{1}\right)=T_{s}+\left(T_{1}-T_{s}\right) \exp \left(-l_{1} / d\right),
$$

which yields

$$
T_{1}=T_{s}+\left(T_{M}-T_{s}\right) \exp \left(+l_{1} / d\right) \text {. }
$$

Assuming constant specific heats, the energy put into the system per unit area is

$$
\begin{aligned}
E_{0}=\left(1-R_{L}\right) E_{p} & =l_{1} L+\int_{0}^{\infty}\left[T(z)-T_{s}\right] C_{p} d z \\
& =l_{1} L+\left(T_{1}-T_{s}\right) C_{p} d
\end{aligned}
$$

where $T_{s}$ is the ambient temperature of the solid, $R_{L}$ is the reflectivity of the liquid, and $L$ is the molar enthalpy change upon melting solid of composition $X_{s}$ at the temperature $T_{i}$ of a superheated interface, which we will approximate by the latent heat of fusion of pure solute at $T_{M}^{\mathrm{A}}$. Combining (23) and (24), we have an expression for $l_{1}$ :

$$
\left(1-R_{L}\right) E_{p}=l_{1} L+\left(T_{M}-T_{s}\right) \exp \left(+l_{1} / d\right) C_{p} d,
$$

which can be solved on a hand calculator for $l_{1}$.

Calculations indicate ${ }^{30,31}$ that the hot liquid cools to nearly uniform temperature ${ }^{38}$ with relatively little interface motion before steady-state regrowth begins. We estimate the time $t_{c}$ necessary for such heat conduction to occur by

$$
\left(2 D_{T} t_{c}\right)^{1 / 2} \approx l_{1} \text {. }
$$

During this time, the temperature profile in the solid broadens. We still approximate it by an exponential curve, but the new decay length is

$$
d^{\prime}=\left[2 D_{T}\left(t_{p}+t_{c}\right)\right]^{1 / 2}=\left(d^{2}+l_{1}^{2}\right)^{1 / 2} .
$$

During rapid regrowth, the interface is significantly undercooled to temperature $T_{i}$. The temperature profile is roughly described by:

$$
\begin{aligned}
& T(z)=T_{i}, \quad 0 \leqslant z \leqslant l_{1}, \\
& T(z)=T_{s}+\left(T_{i}-T_{s}\right) \exp \left[-\left(z-l_{1}\right) / d^{\prime}\right], \quad z>l_{1} .
\end{aligned}
$$

We find the gradient in the liquid at the interface to be essentialy zero and the gradient in the solid at the interface to be

$$
\nabla T_{s i}=-\frac{T_{i}-T_{s}}{\left(d^{2}+l_{1}^{2}\right)^{1 / 2}} .
$$

This temperature gradient remains roughly constant ${ }^{39}$ during steady-state regrowth.

The values of $\nabla T_{s i}$ predicted by Eqs. (25) and (26) are in reasonable agreement with those of Surko et al..$^{30}$ and of Wood and Giles. ${ }^{31}$ Using $L=4192 \mathrm{~J} / \mathrm{cm}^{3}, C_{p}=2.117$ $\mathrm{J} / \mathrm{cm}^{3} \mathrm{~K}, D_{T}=0.7 \mathrm{~cm}^{2} / \mathrm{s}, T_{s}=20^{\circ} \mathrm{C}$, and $R_{L}=0.57$ for comparison with Surko et al. and $R_{L}=0.60$ for comparison with Wood and Giles, the results in Table I are obtained. We have also taken $T_{i}=T_{M}^{\mathrm{S}}=1410^{\circ} \mathrm{C}$ for the purpose of comparison. The resulting gradients are all within a factor of two of the results of the computer calculations, which were taken from Fig. 1 of Ref. 29 and from Fig. 3 of Ref. 30. Consequently, for a good approximate description of regrowth from laser-induced melting, Eq.(22) becomes

$$
D_{T} \nabla T_{s i}=L u / C_{p},
$$

TABLE I. Comparison of predicted temperature profile characteristics with computer calculations.

\begin{tabular}{lllllll}
\hline & $\begin{array}{l}E_{p} \\
\left(\mathrm{~J} / \mathrm{cm}^{2}\right)\end{array}$ & $\begin{array}{l}t_{p} \\
(\mathrm{~ns})\end{array}$ & $\begin{array}{l}d \\
(\mu \mathrm{m})\end{array}$ & $\begin{array}{l}l_{1} \\
(\mu \mathrm{m})\end{array}$ & $\begin{array}{l}d^{\prime} \\
(\mu \mathrm{m})\end{array}$ & $\begin{array}{l}\nabla T_{s i} \\
\left(10^{6} \mathrm{~K} / \mathrm{cm}\right)\end{array}$ \\
\hline Wood and Giles & 1.75 & 25 & - & 0.7 & 3.6 & 3.9 \\
This work & 1.75 & 25 & 1.87 & 0.205 & 1.88 & 7.4 \\
Wood and Giles & 1.25 & 25 & - & 0.45 & 2.1 & 6.6 \\
This work & 1.25 & 25 & 1.87 & 0 & 1.87 & 7.4 \\
Surko et al. & 4.5 & 33 & - & 1.3 & $1.8-5.3$ & $7.7-2.6$ \\
This work & 4.5 & 33 & 2.15 & 1.53 & 2.80 & 5.0 \\
\hline \hline
\end{tabular}


with $\nabla T_{s i}$ given by Eq. (26). Equations (4) or (6), (9), (20), and (27) form a system of four equations which predict laser annealing behavior from knowledge of $E_{p}, t_{\rho}, T_{s}, X_{l}$, and $l$.The remaining unknown is the boundary-layer thickness $l$, which is estimated in the following section.

\section{B. Estimate of boundary-layer thickness for solute diffusion}

Solute transport in the melt has been assumed to be purely diffusive out to a distance $l$ from the interface, beyond which convection effects complete mixing in the liquid. ${ }^{33} \mathrm{~A}$ $1-\mu$ thick layer of laser-melted $\mathrm{Si}$ is five orders of magnitude too thin for natural convection ${ }^{40}$ to set in. Convection can, however, be induced by the variation of surface tension with temperature, by the volume change upon melting and regrowth, ${ }^{42}$ and by nonuniformities in the radiation pressure during melting.

If the last effect dominantes, an estimate of the fluid velocity can be obtained by assuming all of the radiation momentum to be transferred to the fluid. If half of the fluid acquires momentum $p_{f}$ and the other half acquires $-p_{f}$, we have

$$
\left(1+R_{L}\right) E_{p} A / c=p_{f}=\rho u_{0}\left(l_{1} A / 2\right),
$$

where $c$ is the speed of light, $u_{0}$ is the induced fluid speed, $A$ is the area of the molten spot, and $l_{1}$ is the depth of the liquid layer.

The induced convective motion is assumed to be as depicted in Fig. 8. Fluid motion near a point such as point " $S$ " is described by a "stagnation point profile". ${ }^{25}$ The velocity $u$ at a distance $z$ from the interface is equal to $u_{0} / 2$ when

$$
0.5 \approx z\left(\frac{u_{0} \rho}{\eta x}\right)^{1 / 2}
$$

where $x$ is the horizontal distance from $S, \rho$ is the density, and $\eta$ is the viscosity. If we let this define the length $l(x)$ in the neighborhood of stagnation points separated by a typical distance $l_{1}$, then we average across the interface to get

$$
l=\langle l(x)\rangle \approx 0.23\left(\frac{\eta l_{1}}{u_{0} \rho}\right)^{1 / 2} .
$$

For the 33 ns pulse described by Surko et al., $E_{0}=4.5 \times 10^{7}$ $\mathrm{erg} / \mathrm{cm}^{2}$, giving $u_{0} \sim 20 \mathrm{~cm} / \mathrm{s}$ and $l \sim 0.7 \mu$ with an uncertainty of perhaps an order of magnitude. Note in cases where $l$ is greater than $l_{1}$, that the steady-state solution, Eq. (20), to the diffusion equation will only be useful in a qualitative manner. A slightly more sophisticated analysis, perhaps like the "quasi-steady-state" treatment of Liau et al., ${ }^{44}$ may be appropriate. However, whenever sophisticated computer techniques are unavailable or are unnecessarily involved, Eqs. (26) and (28) are probably sufficient.

\section{PRELIMINARY COMPARISON WITH EXPERIMENT}

Equations (4) and (6) for solute trapping cannot be truly tested until $T_{i}, C_{l i}$, and $u$ are either solved for or measured during an experiment. Baeri et $a l .{ }^{45}$ have calculated the latter two variables and measured $C_{s}$ during laser annealing of
$\mathrm{Si}(\mathrm{Bi})$. During the calculation of the growth velocity it is assumed that $T_{i}=T_{M}$ and also that $u$ is independent of $C_{l i}$. These assumptions are questionable; those which lead to Eq (9) seem more appropriate. We can still make a preliminary comparison of the predictions of Eqs. (4) or (6) with such experiments with the following caveats. The theory is only for dilute solutions at this point, since no solute-solute interaction has been considered in the kinetic analysis. In addition, we have no way of knowing $\alpha$ and $D_{i}$ at present; we expect $\alpha=1$ and $D_{i} \approx D_{E} \approx 10^{-5} \mathrm{~cm}^{2} / \mathrm{s}$, which we have chosen for comparison. Instead, we could use them as free parameters to be found by fitting the data. The activity coefficients, $\gamma_{s}$ and $\gamma_{l}$, are at present unknown in $\mathrm{Si}(\mathrm{Bi}), \mathrm{Si}(\mathrm{As})$, and in many other systems of interest; they must be estimated for use in Eq. (9). Finally, the orientation dependence of $f$ (if faceting occurs) or of the ledge height and of $\Delta \mu_{B}^{0 *}$ (if no faceting occurs) has been ignored so far, as has any tendency for solute adsorption or desorption at the interface. On the other hand, the experimental curves and points in Figs. 4 and 5 may appear somewhat different after accounting for the solute effect upon growth velocity and for the effects of an undercooled interface, using Eq. (9), for example. In addition, the tendency for some systems, e.g., $\mathrm{Si}(\mathrm{Bi})$, to phase separate in the liquid state may introduce some uncertainty in the local value of $C_{l i}$.

The data of Baeri et al. are plotted with the theoretical curves in Figs. 4 and 5. The observation of complete trapping of arsenic at very low velocities cannot be explained unless $D_{i}<10^{-3} D_{L}$. The tendency for bismuth trapping to saturate near $k^{\prime}=0.3$ may be explained, if the calculations of $u$ are not significantly in error, in two ways. Either the experiments have demonstrated a kinetic limit to trapping ${ }^{46}$ and we must conclude $\alpha=0.3$ in $\mathrm{Si}(\mathrm{Bi})$ or else $C_{i j}$ has come too close to the $T_{0}$ line and the experiments have run into a thermodynamic limit to solute trapping. In the former case, we expect to see anomalously large vacancy concentrations (or dislocation loops formed by their conglomeration and collapse) in the grown crystal. In the latter case, cutting the bismuth dose should increase the maximum segregation coefficient observed. Accounting for the orientation dependence of $D_{i}$ and of $\lambda$ (note $\lambda_{111} / \lambda_{100} \approx 2.3$ ) will shift the theoretical curves to positions consistent with the orientation dependence of the data.

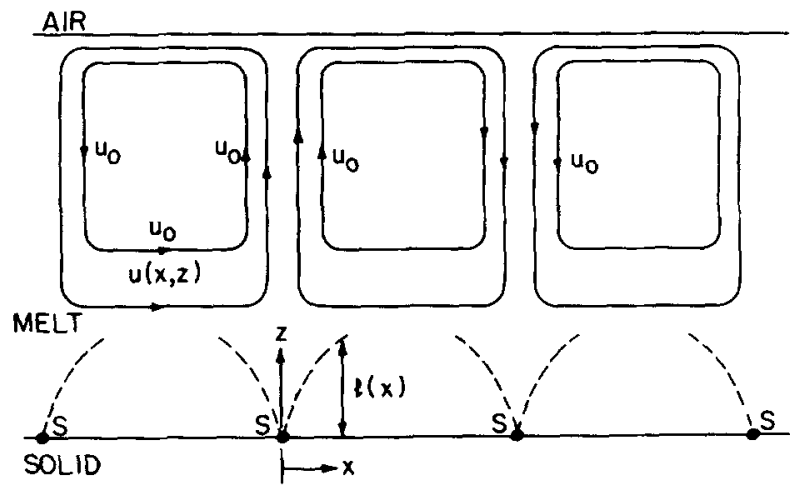

FIG. 8. Assumed convection profile. $l(x)$ is the height at which $u=u_{0} / 2$. 


\section{CONCLUSIONS}

We have developed a microscopic model for solute incorporation at the surface of a rapidly-growing crystal without having to resort to the unknown properties of the solidliquid interface. The results for stepwise growth and for continuous growth have slight quantitative differences but exhibit the same qualitative features. They predict complete solute trapping when growth is more rapid than the diffusive atomic motion of solute trying to escape. The crystal growth process itself is limited only by the impingement frequency of atoms upon the crystal-melt interface and can consequently occur much more rapidly than the solute-solvent redistribution process, with velocities approaching the speed of sound. The location of the transition from equilibrium segregation to complete trapping varies little with equilibrium segregation coefficient, unlike that of Baker. We find a kinetic limit to solute trapping to be quite unlikely, in contrast to the amended model of Jackson, Gilmer, and Leamy, which predicts an absolute maximum of $\left(1+k_{e}\right) / 2$ for the impurity segregation coefficient.

Coupled with macroscopic heat- and solute-diffusion equations, the model is capable of predicting rapid steadystate regrowth velocities and solid compositions from knowledge of the ambient temperature and of the bulk composition of the liquid. With minor modifications, the results can be applied to describe regrowth from laser-induced melting. The temperature profiles so obtained are roughly consistent with computer-generated solutions to the heat equation, where the latter exist. The heat-flow and liquid-diffusion analyses presented here are suggested for use whenever the finite-element results are unavailable or are unnecessarily detailed. The assumptions of no interfacial undercooling and no effect of solute upon the driving force should be replaced by a growth velocity equation such as Eq. (9). Preliminary comparison with experiment indicates the possibility of an unexpectedly low interface diffusivity of As in Si and also the possibility of an unexpected saturation of $\mathrm{Bi}$ trapping in $\mathrm{Si}$.

\section{ACKNOWLEDGMENTS}

The author is grateful to Professor David Turnbull for guidance, to Professor Frans Spaepen for much helpful discussion, and to K. A. Jackson for constructive criticism. This research was supported in part of an Allied Chemical Co. Fellowship Grant and by National Science Foundation Grant DMR-79-23597.

'Complete trapping of $\mathrm{Cu}$ in $\mathrm{Al}\left(k_{\mathrm{e}} \approx 0.17\right)$ was observed by $\mathrm{H}$. Biloni and B. Chalmers, Trans. Metall. Soc. AIME 233, 373 (1965).

${ }^{2}$ J. C. Baker and J. W. Cahn, Acta Metall. 17, 575 (1969).

${ }^{3}$ C. W. White, J. Narayan, and R. T. Young, AIP Conf. Proc. 50, LaserSolid Interactions and Laser Processing - 1978, edited by S. D. Ferris, H. J. Leamy, and J. M. Poate (AIP, New York, 1979), p. 102.

${ }^{4} \mathrm{~K}$. A. Jackson and H. J. Leamy, AIP Conf. Proc. 50, Laser-Solid Interactions and Laser Processing-1978, edited by S. D. Ferris, H. J. Leamy, and J. M. Poate (AIP, New York, 1979), p. 102.

${ }^{5}$ F. Spaepen and D. Turnbull, in Laser and Electron Beam Processing of Semiconductor Structures edited by J. M. Poate and J. W. Mayer (Academic, New York, 1981).

6J. W. Cahn, Acta Metall. 8, 554 (1960).

${ }^{7} \mathrm{~J}$. W. Cahn, W. B. Hillig, and G. W., Sears, Acta Metall. 12, 1421 (1964).
${ }^{8}$ K. A. Jackson, Can. J. Phys. 36, 683 (1958).

${ }^{9}$ Note that the motion indicated by the dashed arrow in Fig. 1 is not so energetically favored as it might at first appear since it leaves behind a vacancy. We must expect $\alpha$ to be large and the fraction of impurity atoms, $1-\alpha$, which are kicked down from a "saddle point" to be quite small. There will be no explicit velocity dependence of $\alpha$, but it may have weak composition dependence and interface temperature dependences (through liquid structure). In any case, $\alpha$ must be unity when the free energy of vacancy formation in the solid monolayer is sufficiently high.

${ }^{10}$ Reported by J. W. Cahn, S. R. Coriell, and W. J. Boettinger, in Laser and Electron Beam Processing of Materials, edited by C. W. White and P. S. Peercy (Academic, New York, 1980), pp. 89-103.

${ }^{11}$ In this analysis, $C_{i i}$ is treated as a constant in time. An analysis where $C_{i i}$ is allowed to vary in time results in an equation coupled to the diffusion equation in the liquid; the results are expected to differ negligibly from those presented here, but the equations are much more difficult to solve.

${ }^{12} \mathrm{Cahn}$, Hillig, and Sears in Ref. 7 suggest the use of $D_{i}=\beta D_{L}$, which will be useful when $\beta$ becomes known for the impurity in question.

${ }^{13}$ J. C. Baker and J. W. Cahn, "Thermodynamics of Solidification," in Solidification (Am. Soc. Metals, Metals Park, Ohio, 1970), pp. 23-58.

${ }^{14}$ R. F. Wood, Appl. Phys. Lett. 37, 302 (1980).

${ }^{15}$ Formally, this is done by integrating $J(z)$ and $C(z)$ between the monolayers.

${ }^{16} \mathrm{H}$. E. Cline, in a paper which has been submitted to this journal, has derived Eq. 6 in a different way.

${ }^{17}$ D. Turnbull and M. H. Cohen, "Crystallization Kinetics and Glass Formation," in Modern Aspects of the Vitreous State, edited by J. D. MacKenzie, 1 (Butterworths, London, 1960),p. 38.

${ }^{18}$ W. B. Hillig and D. Turnbull, J. Chem. Phys. 24, 914 (1956).

${ }^{19}$ D. Turnbull, J. Phys. Chem. 66, 609 (1962).

${ }^{20}$ D. Turnbull, J. Phys. (Paris) 35, C-4, C4.1 (1974).

${ }^{21}$ D. Turnbull and B. G. Bagley, "Transitions in Viscous Liquids and Glasses," in Treatise on Solid State Chemistry, edited by N. B. Hannay (Plenum, New York 1975), Vol. 5, p. 513.

${ }^{22}$ F. Spaepen and D. Turnbull, "Formation of Metallic Glassses," in Rapidly Quenched Metals, 2nd International Conf. edited by N. J. Grant and B. C. Giessen (MIT Press, Cambridge, Massachusetts, 1976), pp. 205-229.

${ }^{23} \mathrm{C}$. V. Thompson and F. Spaepen (to be published). Proceedings of the Materials Research Society Meeting, Boston, November 1981.

${ }^{24}$ The possibility of a new interpretation of $\alpha$ when $T_{i}>T_{0}$ suggests itself. But in this case the formation of vacancies in the solid when $\alpha<1$ will not be necessary as long as growth is diffusion limited.

${ }^{25} \mathrm{M}$. Hillert and B. Sundman, Acta Metall. 25, 11 (1977).

${ }^{26}$ Another unknown, $X_{s}$, appears, coupling Eq. (9) back to (4) or (6) and making the system more difficult to solve.

${ }^{27}$ Hillert and Sundman in Ref. 25 make the opposite assumption. Neither assumption may be necessary; the development of a means of explicitly accounting for both dissipative processes is in progress.

${ }^{28} \mathrm{~K}$. A. Jackson, G. H. Gilmer, and H. J. Leamy, in Laser and Electron Beam Processing of Materials, edited by C. W. White and P. S. Peercy (Academic, New York, 1980), pp. 104-110.

${ }^{29} \mathrm{~A}$ different choice of $\Delta \mu_{\mathrm{A}}^{0 *}$ and $\Delta \mu_{\mathrm{B}}^{0 *}$ is better if we want to bring this treatment into agreement with the aforementioned basic difference between the crystallization process and the solute-solvent exchange process. To realistically describe alloy solidification, we expect $\Delta \bar{G}^{0 *}$ (crystallization $) \approx 0$ and $\Delta \bar{G}^{0}$ (exchange $)=-R T_{i} \ln \left(D_{i} / \lambda^{2} f v \gamma_{s}\right)$. Now the value of the former molar free energy of activation will be determined mainly by the value of $\Delta \mu_{\mathrm{A}}^{0 *}$ (crystallization) because all of the atoms, the vast majority of which are solvent, crystallize. The value of the latter molar free energy of activation, that for solute-solvent exchange, should be essentially determined by the value of $\Delta \mu_{\mathrm{B}}^{\mathrm{O}}$ (exchange), since we only find a distinguishable exchange when a $\mathbf{B}$ atom is involved and because the minor component rarely exchanges with itself. Hence, we may not err too badly by letting $J^{A}$ take care of the growth velocity (which is similar to our treatment in the previous section) and letting $J^{\mathrm{B}}$ take care of solute-solvent exchange. This is done by taking Eq. $(17)$ with $0=\Delta \mu_{\mathrm{A}}^{0_{*}}$ and $D_{i}=\lambda^{2} f v \gamma_{s} \exp \left(-\Delta \mu_{\mathrm{B}}^{0 *} / R T_{i}\right)$, resulting in $J_{i \rightarrow s}^{\mathrm{A}} / C_{i i}^{\mathrm{A}}=\lambda f v \approx f u_{s}$, where $u_{s}$ is the speed of sound. Equation (14) becomes

$u \approx f u_{s}\left[1-\exp \left(\Delta \mu_{\mathrm{A}}^{0 *} / R T_{i}\right)\right]$ and $(17)$ becomes once again identical to (6). In this case, however, the entire velocity range $u<u_{s}$ is accessible; there is no kinetic limit to solute trapping.

${ }^{30}$ C. M. Surko, A. L. Simons, D. H. Auston, J. A. Golovchenko, and R. E. Slusher, AIP Conf. Proc. 50, Laser-Solid Interactions and Laser Processing-1978, edited by S. D. Ferris, H. J. Leamy, and J. M. Poate (AIP, New 
York, (1979), p. 155.

${ }^{31}$ R. F. Wood and G. E. Giles, Phys, Rev. B 22, 2923.

${ }^{32}$ C. G. Levi and R. Mehrabian, ONR Technical Report NO0014-78-C$0275(1980)$.

${ }^{33}$ J.A. Burton, R. C. Prim, and W. P. Slichter, J. Chem. Phys. 21, 1987 (1953).

${ }^{34} \mathrm{G}$. C. Levi and R. Mehrabian, ONR Technical Report N00014-78-C0275 (1980), Figs. 2, 6, 7, and 33 .

${ }^{35} \mathrm{~N}$. Bloembergen, AIP Conf. Proc. 50 Laser-Solid Interactions and Laser Processing - 1978, edited by S. D. Ferris, H. J. Leamy, and J. M. Poate (AIP, New York, 1979), p. 1.

${ }^{36}$ This appears credible in view of Fig. 1 of Ref. 29.

${ }^{37}$ More precisely, $T_{0}\left(X_{s}\right)$. Actually, the latter two assumptions cannot be exact in view of Eqs. (9) and (22), but we can do no better without resorting to intricate computer programs. The errors introduced should have relatively little effect on the final result, which is a number for $\nabla T_{s i}$.

${ }^{38}$ In the calculations of Wood and Giles, reported values of $\nabla T_{l i}$ indicate that during the steady-state regrowth period, the temperature difference across the liquid layer is less than $5^{\circ} \mathrm{C}$.

${ }^{34}$ According to this analysis, the gradient should continue to decrease as $t^{-1 / 2}$. An average over the appropriate part of the regrowth period could be taken if necessary.

"There are two types of convection: "natural," or thermal-gradient-induced, and "forced." The former can be shown not to occur (see Ref. 40) if the Rayleigh number, a dimensionless combination including the thermal expansion coefficient, viscosity, thermal conductivity, and the cube of the depth of the liquid layer, falls below a critical value which is of order $10^{3}$.

${ }^{41}$ L. A. Segel, "Non-liner Hydrodynamic Stability Theory and its Applications to Thermal Convection and Curved Flows," in Non-Equilibrium Thermodynamics: Variational Techniques and Stability edited by R. J. Donnelly, R. Herman, and I. Prigogine (University of Chicago, Chicago, 1966), p. 165.

${ }^{42}$ R. A. Brown (private communication).

${ }^{43} \mathrm{H}$. Schlichting, Boundary Layer Theory, 6th ed. (McGraw-Hill, New York, 1968), Chap. 7.

${ }^{44}$ Z. L. Liau, B. Y. Tsaur, S. S. Lau, I. Golecki, and J. W. Mayer, AIP Conf. Proc. 50, Laser-Solid Interactions and Laser Processing-1978, edited by S. D. Ferris, H. J. Leamy, and J. M. Poate (AIP, New York, 1979), p. 105. ${ }^{45}$ P. Baeri, G. Foti, J. M. Poate, S. V. Campisano, and A. G. Cullis, Appl. Phys. Lett. 38, 800 (1981).

${ }^{46}$ Recent indication that this is probably not the case is given in the measurements by White et $a l^{47}$ of $\mathrm{SiBi}\langle 100\rangle$ trapping at $\left(u, k^{\prime}\right)=4.5 \mathrm{~m} / \mathrm{s}$, $0.35)$ and $(6 \mathrm{~m} / \mathrm{s}, 0.45)$, contradicting the reported observations of "saturation" and fitting the stepwise growth model remarkably well.

${ }^{47}$ C. W. White, B. R. Appleton, B. Stritzker, D. M. Zehner, and S. R. Wilson in Laser and Electron Beam Solid Interactions and Materials Processing, edited by J. F. Gibbons, L. D. Hess, and T. W. Sigmon (North Holland, New York, 1981), p. 59. 